\title{
miR-20a-5p promotes adipogenic differentiation of murine bone marrow stromal cells via targeting Kruppel-like factor 3
}

\author{
Endong Zhu, Juanjuan Zhang, Jie Zhou, Hairui Yuan, Wei Zhao and Baoli Wang \\ Key Laboratory of Hormones and Development (Ministry of Health), Tianjin Key Laboratory of Metabolic Diseases, Tianjin Metabolic Diseases Hospital \& \\ Tianjin Institute of Endocrinology, Tianjin Medical University, Tianjin, China \\ Correspondence should be addressed to B Wang: blwang@tmu.edu.cn
}

\begin{abstract}
miR-20a-5p has recently been identified to induce adipogenesis of established adipogenic cell lines in our previous study. However, its role and molecular mechanisms in the regulation of adipocyte lineage commitment of bone marrow-derived stromal cells (BMSCs) still need to be explored. In this report, we demonstrated the expression of miR-20a-5p was promoted gradually during adipogenic differentiation in BMSCs. We also confirmed that miR-20a-5p has a positive function in the adipogenic differentiation of BMSCs by gain-of-function study with overexpression lentivirus or synthetic mimics of miR-20a-5p, and loss-of-function study with sponge lentivirus or synthetic inhibitor of miR-20a-5p. Dual luciferase reporter assay, GFP repression assay and Western blotting suggested Kruppel-like factor 3 (KIf3) was a direct target of miR-20a-5p. Furthermore, siRNA-mediated silencing of $K I f 3$ recapitulated the potentiation of adipogenesis induced by miR-20a-5p overexpression, whereas enhanced expression of $K / f 3$ attenuated the effect of miR-20a-5p. As KIf3 was reported to play an inhibitory role in adipogenesis at the initial stage of differentiation, the findings we present here indicate that miR-20a-5p promotes adipocyte differentiation from BMSCs by targeting and negatively regulating KIf3 in the early phase during the procedure of adipogenesis.
\end{abstract}

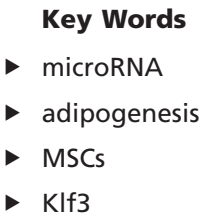

Journal of Molecular Endocrinology (2018) 60, 225-237

\section{Introduction}

Bone marrow-derived stromal cells (BMSCs) in culture can differentiate into several distinct cell lineages, including adipocytes, osteoblastes or chondrocytes (Pittenger et al. 1999, Caplan \& Brude 2001). There are two major phases during the procedure of adipogenesis in vitro: the first phase was named as determination or commitment, which involves the conversion of the stem cell to pre-adipocyte; the second phase was termed as terminal differentiation, which results in the maturation of adipocyte from the pre-adipocyte (Rosen \& MacDougald 2006). Adipocyte differentiation is a well-orchestrated and multistep process that requires the sequential activation of signaling pathways and expression of numerous transcription factors. In general, CCAAT/enhancer-binding protein (C/EBP) $\beta$ and C/EBP $\delta$ are activated very early during adipogenesis, which transcriptionally activates the expression of low levels of $\mathrm{C} / \mathrm{EBP} \alpha$ and peroxisome proliferator-activated receptor $\gamma$ (PPAR $\gamma)$, and both of the latter factors can then induce each other's expression in a positive feedback loop (Yeh et al. 1995, Lefterova et al. 
2008). Finally, many downstream target genes are induced specially for promoting and maintaining the differentiated state of the adipocyte such as adipocyte protein 2 (aP2) (Tang \& Lane 2012).

Adipogenesis is also regulated by lots of other transcription factors, for instance, the Kruppel-like factor (KLF) family (Sarjeant \& Stephens 2012). KLFs are a large family of zinc-finger transcription factors, and it is derived from the drosophila protein 'Kruppel', which shares homology of proteins to the DNA-binding domains (Zuo et al. 1991). KLFs are critical in many physiological and pathological processes including cell proliferation, differentiation, inflammation and apoptosis. Recently, many KLF members have been identified to be involved in both adipogenesis and lipogenesis (Wu \& Wang 2013). Klf3, as a member of the KLF family, could directly bind to the endogenous C/ebpa promoter and repress its activity, and overexpression of Klf3 resulted in decreased adipogenesis from 3T3-L1 cells (Sue et al. 2008).

microRNAs (miRNAs) are $\sim 22$ nucleotides non-coding RNAs that function as guide molecules in RNA silencing by base pairing with its target mRNAs (Ha \& Kim 2014). miRNAs are involved in nearly all developmental and pathological processes in animals, and recent emerging evidences suggest that miRNAs have the capacity to regulate adipogenesis by suppressing various target genes associated with adipogenic differentiation. For instance, miR-17-92 cluster, miR-204, miR-30e, miR-223 and miR-148a-3p promote adipocyte differentiation by negatively regulating retinoblastoma family $(\mathrm{pRb})$ gene $\mathrm{Rb} 2 / \mathrm{p} 130$, runt-related transcription factor 2 (Runx2), low-density lipoprotein receptor-related protein 6 (Lrp6), fibroblast growth factor receptor 2 ( $F g f r 2)$ and lysinespecific demethylase $6 \mathrm{~b}(K d m 6 b)$ respectively (Wang et al. 2008, 2013, Huang et al. 2010, Guan et al. 2015, Tian et al. 2017). miRNA let-7, miR-448, miR-24, miR-302a and miR-215 impair adipocyte differentiation by suppressing high-mobility group AT-hook 2 (Hmga2), Klf5, fatty acid-binding protein 4 (Fabp4, also termed aP2), PPAR $\gamma$, fibronectin type III domain-containing 3B $(F n d c 3 b)$ and catenin, beta-interacting protein 1 (Ctnnbip1) respectively (Sun et al. 2009, Kinoshita et al. 2010, Kang et al. 2013, Jeong et al. 2014, Peng et al. 2016).

miR-20a-5p is a member of miR-17-92 family, we have previously demonstrated that miR-20a-5p was induced in primary cultured bone marrow stromal cells and established adipogenic cell lines after adipogenic treatment (Zhou et al. 2015). We also found supplementing miR-20a-5p could enhance adipogenesis in mouse adipogenic cell lines such as 3T3-L1 preadipocytes, ST2 stromal cells and C3H10T1/2 mesenchymal cells. However, the effects of miR-20a-5p on adipogenic differentiation in BMSCs were not investigated, and the exact mechanisms by which miR-20a-5p determines the adipogenic fates of BMSCs is not yet been clarified.

In current study, we found the expression of miR-20a-5p was kept in a high level during adipogenic differentiation. Furthermore, we show that miR-20a-5p plays a positive role in the regulation of adipogenic differentiation in BMSCs by targeting Klf3. Our findings shed new light on the mechanisms of miR-20a-5p in adipogenesis.

\section{Materials and methods}

\section{Ethics statement, mice}

Four-week-old male C57BL/6J mice were purchased from HFK bioscience company (Beijing, China). All animal experiments and procedures were approved by the Animal Ethical and Experimental Committee of the Tianjin Medical University Metabolic Diseases Hospital.

\section{Cells culture}

BMSCs were isolated from femurs and tibias of 4-week-old C57BL/6J mice and cultured in $\alpha$-MEM supplemented with $10 \%$ fetal bovine serum (FBS) in $10 \mathrm{~cm}$ dish as previously described (Zhou et al. 2017). When cells reached about $90 \%$ confluences, they were cultured in adipocyte-inducing medium (AIM, $\alpha$-MEM containing 10\% FBS, $0.5 \mathrm{mM}$ dexamethasone, $\quad 0.25 \mathrm{mM}$ methylisobutylxanthine, $5 \mathrm{mg} / \mathrm{mL}$ of insulin, and $50 \mathrm{mM}$ indomethacin) for $72 \mathrm{~h}$, followed by treatment for an additional $48 \mathrm{~h}$ with $5 \mathrm{mg} / \mathrm{mL}$ insulin alone. Human embryonic kidney (HEK)-293T cells were cultured in DMEM (Hyclone, ThermoFisher) with $10 \%$ FBS. All cells were maintained in a humidified incubator containing $5 \% \mathrm{CO}_{2}$ at $37^{\circ} \mathrm{C}$.

\section{Reagents}

For Western blot assay, anti- $\beta$-actin was purchased from Sigma Chemical, anti-C/EBP $\alpha$, anti-PPAR $\gamma$ and anti-aP2 were purchased from Cell Signaling Technology; antiKLF3 was purchased from Abcam and anti-KLF5 and antiKLF7 were purchased from Protein Tech Group Ptglab (Wuhan, China). Lipofectamine RNAiMAX was purchased from Invitrogen. 


\section{Quantitative RT-PCR}

Total RNA was extracted from cells using a total RNA isolation kit (Omega BioTek, Norcross, GA, USA). $0.5 \mu \mathrm{g}$ total RNA of each sample was reverse-transcribed (RT) to cDNA with Reverse Transcription Kit (Thermo Fisher). Quantitative PCR (qPCR) analyses for the mRNA expression of $C / e b p \alpha, P p a r \gamma, a P 2$ and $K l f 3$ were performed by using SGExcel Fast SYBR Mixture kits (Sangon Biotech, Shanghai, China) on the Light Cycler 96 Real-Time PCR System (Roche Diagnostics), the mRNA level of NONO was used as an internal control (Arsenijevic et al. 2012), and the detail parameters was followed as previously described (Zhu et al. 2016). For the expression analysis of miR-20a-5p, $0.5 \mu$ g RNA were reverse-transcribed to cDNA using the specific RT primer. Subsequently, miR-20a-5p was PCR amplified on a real-time PCR system using specific forward primers and a universal reverse primer, its expression level was normalized to U6, the detail parameters was followed as previously described (Zhou et al. 2015). All relative expression was calculated using

Table 1 Sequences of oligonucleotides used in this article.

\begin{tabular}{l} 
Name \\
\hline Primer for stem-loop qRT-PCR studies to \\
miR-20a-5p-RT \\
U6-RT \\
miR-20a-5p-specific F \\
U6-specific F \\
Universal R \\
Primer for qRT-PCR analyses to mRNA \\
Clebp $\alpha$-F \\
Clebp $\alpha-R$ \\
PPAR $-\mathrm{F}$ \\
PPAR \\
aP2-R \\
aP2-R \\
KIf3-F \\
KIf3-R \\
Nono-F \\
Nono-R \\
Ectopic expression vector construction \\
KIf3-F \\
KIf3-R \\
Report vector construction \\
KIf3-3'UTR-F \\
KIf3-3'UTR-R \\
KIf3-3'UTR-MUT-F \\
KIf3-3'UTR-MUT-R \\
Lentivirus vector construction \\
Pre-miR-20a-5p-F \\
Pre-miR-20a-5p-R \\
miR-20a-5p-sponge-F \\
miR-20a-5p-sponge-R \\
miR-20a-5p mimics-sence \\
miR-20a-5p mimics-anti-sence \\
mimics NC-sence \\
inhibitor NC \\
siRNA sequences \\
siKIf3-1 \\
\hline
\end{tabular}

Sequence

miRNA

5'-GTC GTA TCC AGT GCA GGG TCC GAG GTA TTC GCA CTG GAT ACG ACC TAC CTG CAC TAT A-3'

5'-GTC GTA TCC AGT GCA GGG TCC GAG GTG CAC TGG ATA CGA CAA AAT ATG G-3'

5'-ATG CTA AAG TGC TTA TAG T-3'

5'-TGC GGG TGC TCG CTT CGG C-3'

5'-CCA GTG CAG GGT CCG AGG T-3'

5'-CTG ATT CTT GCC AAA CTG AG-3'

5'-GAG GAA GCT AAG ACC CAC TAC-3'

5'-CTT GAC AGG AAA GAC AAC GG-3'

5'-GCT TCT ACG GAT CGA AAC TG-3'

5'-AAA TCA CCG CAG ACG ACA GG-3'

5'-GGC TCA TGC CCT TTC ATA AAC-3'

5'-GAA CCA TCC TTC CGT CAT CG-3'

5'-TCA TCA GAC CGA GCG AAC TT-3'

5'-TGC TCC TGT GCC ACC TGG T-3'

5'-CCG GAG CTG GAC GGT TGA AT-3'

5'-CGG GAT CCG CCA GGA TGC TCA TGT TTG ATC CAG TCC-3'

5'-GCT CTA GAT CAG ACT AGC ATG TGG CGT TTC C-3'

5'-CGA GCT CGT CTT GAA GGA GTT GCT AAC G-3'

5'-CCC AAG CTT AAT GCA ATA AGC CTG CTA TG-3'

5'-CAA ATT CAA CCT CAT ATT TTT CCT GTT ATG TAC CTA GTG TTA GAG GG-3'

5'-AAA AAT ATG AGG TTG AAT TTG TAA AAC ACA TTG TAG CCC TCT T-3'

5'-AGA ATT CTG TCG ATG TAG AAT CTG CCT G-3'

5'-AGG ATC CGA CAG TTT GAT TGG GCG ACA G-3'

5'-TGC ACT ATA AGC ACT TTA GCT ACC TGC ACT ATA AGC ACT TTA GCT ACC TGC ACT

ATA AGC ACT TTA TTT TTT ACG CGT GAA TTC ACT GGC CGT CGT T-3'

5'-GGT AGC TAA AGT GCT TAT AGT GCA GGT AGC TAA AGT GCT TAT AGT GCA GGT AGC TAA AGT GCT TAT AGT GCA GGT AGC GGA TCC TCT AGA GTC GAC CT-3'

5'-CCCGUCGAAUUACAUAGAA-3'

5'-CCAGUACCUGUAAUUGAAU-3'

sfection

5'-UAA AGU GCU UAU AGU GCA GGU AG-3'

5'-ACC UGC ACU AUA AGC ACU UUA UU-3'

5'-CUA CCU GCA CUA UAA GCA CUU UA-3'

5'-UUC UCC GAA CGU GUC ACG UTT-3'

5'-ACG UGA CAC GUU CGG AGA ATT-3'

5'-CAG UAC UUU UGU GUA GUA CAA-3'

F, forward; NC, negative control; R, reverse. 
the comparative threshold cycle method. The primer sequences used in the study were listed in Table 1.

\section{Construction of plasmids, lentivirus packaging and transduction}

For lentivirus-mediated overexpression of miR-20a-5p, genomic sequence spanning the mouse miR-20a-5pcoding region flanked by approximately 100 base pairs from $5^{\prime}$ or $3^{\prime}$ on either end was cloned into lentiviral vector pCDH-CMV-MCS-EF1-copGFP (CD511B-1, System Bioscience, Mountain View, CA, USA) between the EcoR I site and the BamH I site. For control, the lentivirus packaged with the empty vector.

miR-20a-5p sponge vector was constructed as follows: the fragment containing 6 copies of miR-20a-5p-binding sites was amplified by PCR from pUC19 vector with two long specific primers, and then, this fragment was cloned into the pLVX-shRNA2 vector (Clontech Laboratories) at the BamH I site and the EcoR I site. The lentivirus packaged with the empty pLVX-shRNA2 vector served as a control. Virus was produced and target cells were infected according to the user's manual.

The construction of report vectors was based on the pMIR-REPORT and pMIR-GFP-REPORT for miR-20a-5p targets. The 3'-UTR mRNA sequences of Klf3 containing the miR-20a-5p-binding site were amplified by PCR, and PCR products were cloned into vectors at Spe I and Hind III sites. Moreover, the mutant report vectors was constructed by In-Fusion cloning technology using Mut Express II Fast Mutagenesis Kit V2 (Vazyme, Nanjing, China) according to the user's manual.

The coding sequence of Klf3 was amplified by PCR and cloned into pCDNA3.1 (+) vector at BamH I and $\mathrm{Xba}$ I sites.

The sequences of primers used are shown in Table 1.

\section{Western blotting}

Treated cells were lysed by RIPA Lysis Buffer (Beyotime, Shanghai, China), and the immunoblotting was performed as previously described (Zhu et al. 2014). Briefly, the protein was separated in $12 \%$ SDS denatured polyacrylamide gel and then transferred onto a polyvinylidene difluoride membrane. The membranes were blocked with 5\% skim milk at room temperature for $1 \mathrm{~h}$ and were incubated with appropriate antibodies at $4^{\circ} \mathrm{C}$ overnight respectively. Membranes were washed and incubated with horseradish peroxidase (HRP)-conjugated secondary antibodies (1:3000, TransGen Biotech, Beijing, China) according to the manufacturer's instructions. Finally, the protein of interest was visualized using Immobilon Western Chemiluminescent HRP Substrate (Millipore).

\section{Oil red $O$ staining}

Treated adipocyte-inducing BMSCs were gently washed twice with cold $1 \times$ PBS, and then fixed in $4 \%$ paraformaldehyde for $10 \mathrm{~min}$. Next, the cells were washed twice with deionized water, and then stained with $60 \%$ saturated oil red $\mathrm{O}$ for $5 \mathrm{~min}$. For oil red $\mathrm{O}$ quantification, isopropanol was added to each well, and then the plate was rocked on a shaker for $15 \mathrm{~min}$. Light absorbance by the extracted dye was measured at $520 \mathrm{~nm}$.

\section{Cell transfection}

Transfection was performed with Lipofectamine RNAiMAX Reagent according to the manufacturer's protocol. Briefly, cells were seeded in plates the day before transfection to ensure a suitable cell confluent on the day of transfection. For transfection of the oligonucleotides, $50 \mathrm{nM}$ miR-20a-5p mimics, mimics negative control RNA (miR-NC), inhibitor and inhibitor NC (GenePharma, Shanghai, China) were used. For plasmids, transfection was performed with Lipofectamine 2000 Reagent, 80 ng of each firefly luciferase reporter vector, $20 \mathrm{ng}$ of Renilla luciferase control vector and $200 \mathrm{ng}$ GFP report vectors were used.

\section{Luciferase assay and GFP repression experiments}

HEK-293T cells were seeded in a 96-well plate, and 5000 cells were placed in each well the day before transfection. The cells were transfected with each firefly luciferase reporter vector, Renilla luciferase control vector pRL-TK (Promega), and either miR-20a-5p mimics or miR-NC for $48 \mathrm{~h}$. The luciferase assay was performed as described (Zhu et al. 2014).

For GFP repression experiments, HEK-293 cells were seeded in a 12 -well plate at $1 \times 10^{5}$ cells per well the day before transfection, and then were co-transfected with either miR-20a-5p mimics or miR-NC, and various GFP reporter vectors for $48 \mathrm{~h}$. The cells were analyzed by fluorescence microscope and flow cytometer as described previously (Zhu et al. 2014). 


\section{Statistical analysis}

The results are expressed as mean \pm standard deviation (s.D.). A two-tailed Student's $t$ test was applied to analyze the differences between the two groups, and one-way ANOVA was used followed by Tukey's test to analyze the differences between multiple groups. Statistical analysis was performed with SPSS software (version 17). Statistical differences were declared significant at $P<0.05$ level. Statistically significant data are indicated by asterisks $\left(P<0.05\left(^{*}\right), P<0.01\left(^{* *}\right)\right.$, and $\left.P<0.001{ }^{(* *}\right)$ ).

\section{Results}

\section{miR-20a-5p was upregulated during adipocyte differentiation in BMSCs}

We have found that miR-20a-5p was increased in BMSCs at $72 \mathrm{~h}$ after adipogenic treatment and demonstrated that supplementing miR-20a-5p activity favored adipogenic differentiation from preadipocyte line 3T3-L1, stromal cell line ST2 and mesenchymal cell line C3H10T1/2 (Zhou et al. 2015). In this study, to further define the function and mechanism of miR20a-5p in adipogenic differentiation, we switched to the primary cultured BMSCs cell system. We monitored the expression of miR-20a-5p during adipocyte differentiation in BMSCs. Total RNA was collected at various stages throughout BMSCs differentiation and analyzed by RT-qPCR. As expected, the relative expression of adipogenic differentiation marker genes C/ebpa, Ppary and aP2 was induced in BMSCs after adipogenic treatment (Fig. 1A, B and C). The expression of miR-20a-5p was upregulated gradually for the first 3 days, and its expression remained elevated at following 2 days compared to day 0 (Fig. 1D). Based on these results, we speculated that miR-20a-5p might play a regulatory role in BMSCs adipogenic differentiation.

\section{Overexpression of miR-20a-5p enhanced adipocyte formation in BMSCs}

To certify the role of miR-20a-5p during adipocyte differentiation from BMSCs, we made lentiviral constructs for miR-20a-5p manipulation in vitro. We constructed a plasmid-encoding pre-miR-20a-5p and used the empty vector as NC. Lentiviruses were packaged and named as LV-miR and LV-miR-ctrl respectively, and then the BMSCs were infected with LV-miR-20a-5p or LV-miR-ctrl for 2 days. The increased expression of miR-20a-5p was confirmed by RT-qPCR (Fig. 2A). Following adipogenic
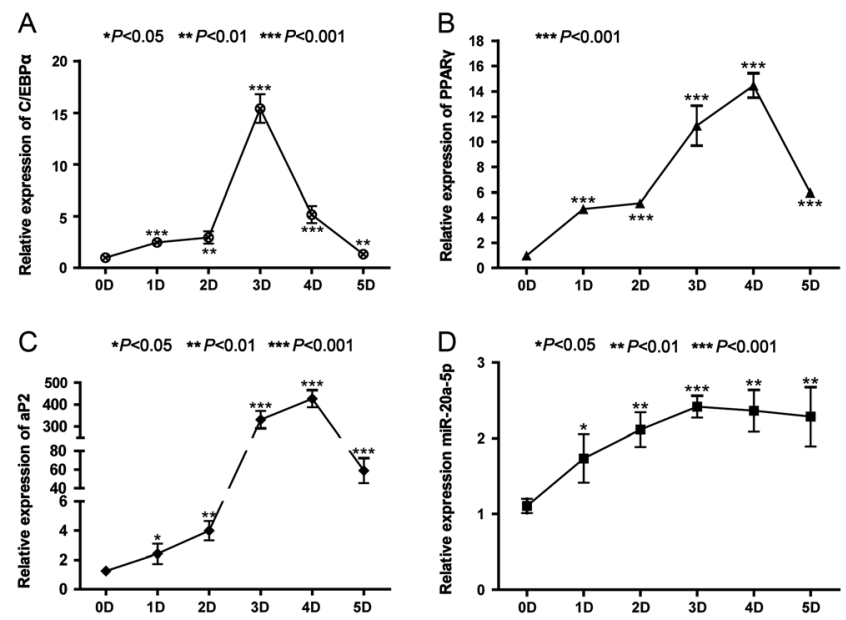

Figure 1

miR-20a-5p was upregulated during adipocyte differentiation in BMSCs. Total RNA was extracted from BMSCs at the indicated time points after induction of adipogenic differentiation. (A, B and C) The mRNA expression levels of C/ebp $\alpha, P p a r \gamma$ and $a P 2$ were measured by RT-qPCR and normalized to Nono levels. (D) The expression levels of miR-20a-5p was tested by RT-qPCR and normalized to U6 level. The levels of the measurements at day 0 were set as 1 . All data are representative of three independent experiments. The values are represented as means \pm S.D. $(n=3)$. ${ }^{*} P<0.05, * * P<0.01, * * * P<0.001$, compared with the day 0 group (ANOVA followed by Tukey's test was applied).

treatment, the miR-20a-5p expressing virus led to a significant increase of differentiated adipocyte numbers (28\% increases in oil red O staining compared to LV-miRctrl group) (Fig. 2B and C). Accordingly, the protein levels of PPAR $\gamma, \mathrm{C} / \mathrm{EBP} \alpha$ and aP2 were significantly increased 3 days after adipogenic treatment (Fig. 2D). Furthermore, BMSCs were transfected with miR-20a-5p mimics to supplement the activity of miR-20a-5p, and the validity of miR-20a-5p ectopic expression was confirmed by RT-qPCR (Fig. 2E). We further demonstrated that miR-20a-5p mimics also significantly promoted adipogenic differentiation from BMSCs (26\% increases in oil red O staining compared to miR-NC group) (Fig. 2F and $\mathrm{G})$. Consistent with this, enhanced expression of PPAR $\gamma$, C/EBP $\alpha$ and aP2 was confirmed by Western blotting analysis (Fig. 2H). These results reveal that overexpression of miR-20a-5p can promote the adipogenic differentiation from BMSCs.

\section{Inhibition of miR-20a-5p suppressed adipocyte formation in BMSCs}

To further verify the promotion of adipogenesis by miR-20a-5p, loss-of-function analysis was performed using miR-20a-5p sponge lentivirus (LV-sponge). Dramatically, inhibition of miR-20a-5p attenuated the adipocyte 
A

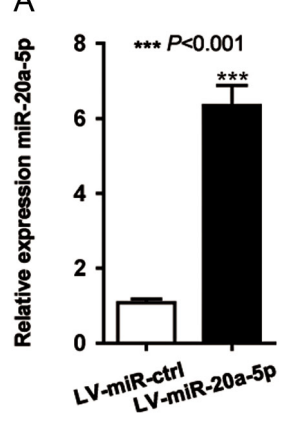

B
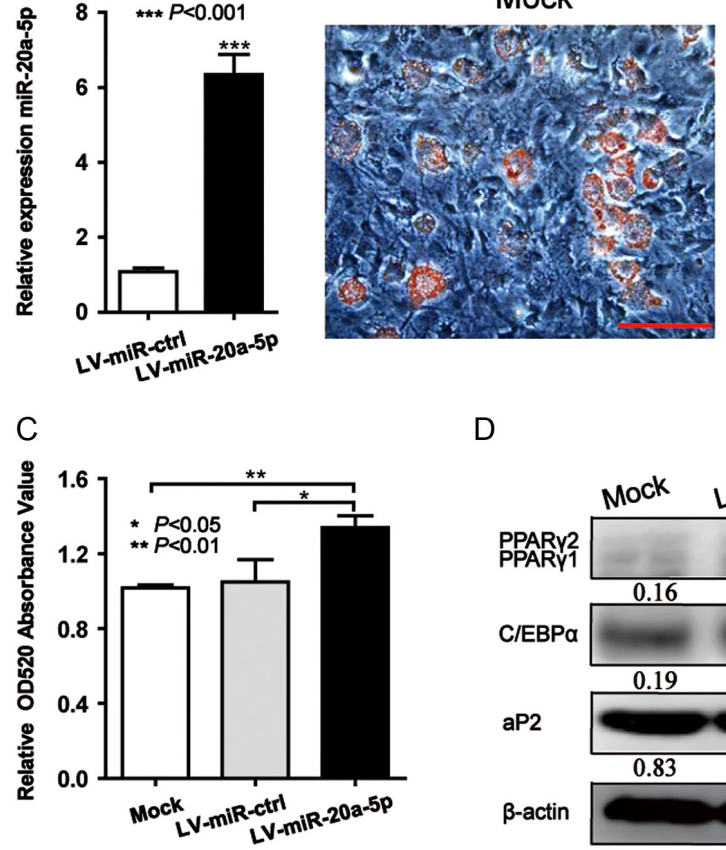

D

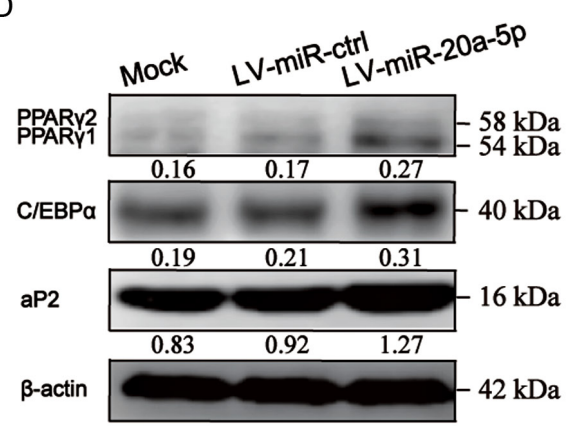

LV-miR-ctrl

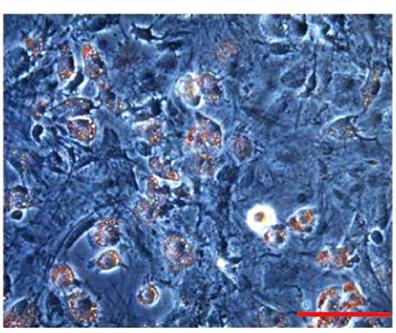

LV-miR-20a-5p

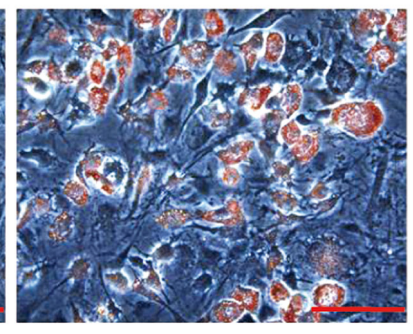

\section{E}

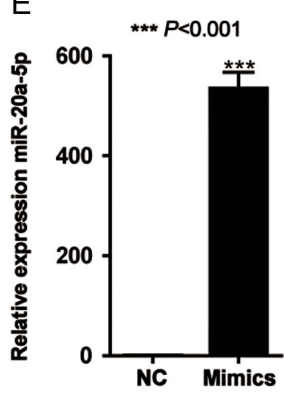

F

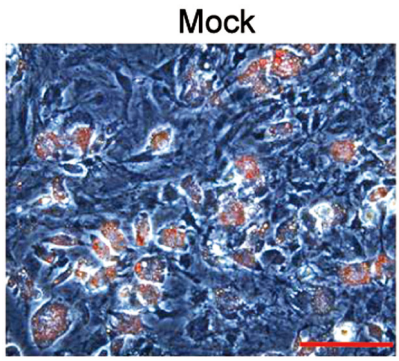

G

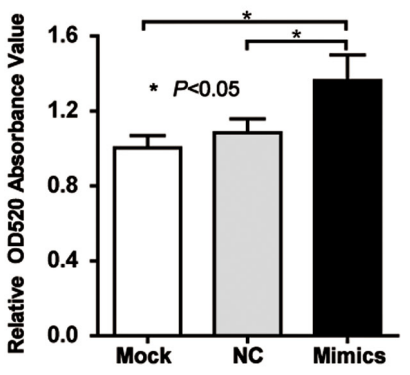

NC

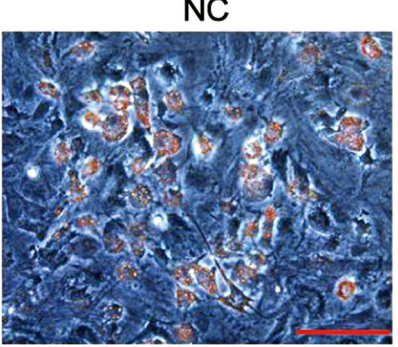

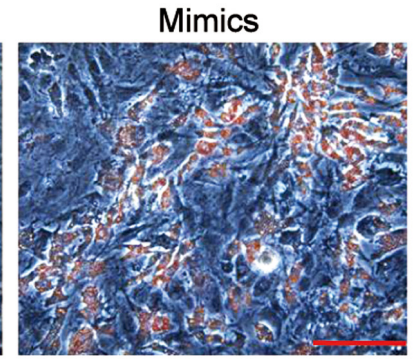

$\mathrm{H}$

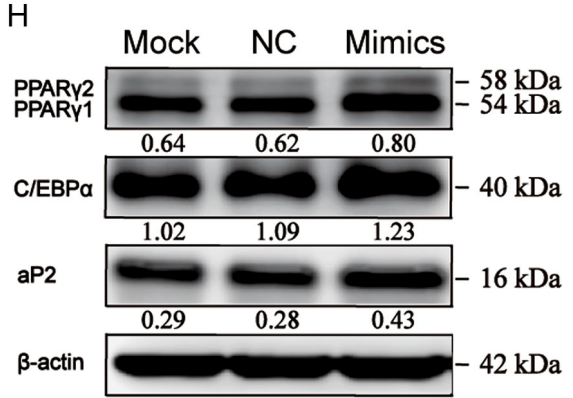

Figure 2

Overexpression of miR-20a-5p enhanced adipocyte formation from BMSCs. (A) Relative expression of miR-20a-5p was measured by RT-qPCR in BMSCs infected with pre-miR-20a-5p-encoding lentivirus (LV-miR-20a-5p) or its NC lentivirus (LV-miR-ctrl) for 2 days. (B, C and D) BMSCs were infected with lentivirus for 2 days, and then cultured in adipocyte-inducing medium (AIM). After 5 days, the adipocyte-inducing BMSCs were stained with Oil Red O, and representative images were shown (B). Oil Red O extracted with isopropanol was measured at OD520 (C). After 3 days, the adipocyte-inducing BMSCs were harvested, and the protein levels of PPAR $\gamma, C / E B P \alpha$ and aP2 were tested by Western blot analysis (D). (E) Relative expression of miR-20a-5p was measured by RT-qPCR in BMSCs transfected with miR-20a-5p mimics or its negative control (NC) for 2 days. (F, G and H) BMSCs were transfected with miR-20a-5p mimics or NC for 2 days, and then cultured in AIM. After 5 days, the adipocyte-inducing BMSCs were labeled with Oil Red O, and representative images were shown (F). Oil Red O extracted with isopropanol was measured at OD520 (G). The protein levels of PPAR $\gamma$, C/EBP $\alpha$ and aP2 were analyzed by Western blotting 3 days after adipogenic treatment $(\mathrm{H})$. All data above represent three separate experiments. All photographs were taken using a light microscope at $200 \times$ magnification, and the scale bars are presented at $100 \mu \mathrm{m}$. The value in $\mathrm{D}$ and $\mathrm{H}$ under each lane indicates the relative expression level of the putative target gene, which is represented by the intensity ratio between PPAR $\gamma, \mathrm{C} / \mathrm{EBP} \alpha$ or aP2 and $\beta$-actin bands in each lane; $\beta$-actin was used as the internal control. The values are mean \pm S.D. $(n=3), * P<0.05, * * P<0.01, * * * P<0.001$, presented relative to NC group (Student's $t$ test was applied in A and E, and ANOVA followed by Tukey's test was applied in C and G). 
formation from BMSCs, and oil red O quantification revealed a $27 \%$ decrease after LV-sponge infection compared to control LV-sponge infection (LV-spongectrl) (Fig. 3A and B). Immunoblotting further confirmed decreased protein expression levels of PPAR $\gamma, \mathrm{C} / \mathrm{EBP} \alpha$ and aP2 (Fig. 3C). Next, the effect of miR-20a-5p antisense oligonucleotide inhibitor was examined on adipogenesis from BMSCs. As shown in Fig. 3D, the expression level of miR-20a-5p was significantly reduced by this inhibitor. Consistently, repressed expression of miR-20a-5p through its inhibitor could reduce the adipogenic differentiation from BMSCs (30\% decrease in oil red O staining compared to inhibitor NC group) (Fig. 3E and F). Furthermore, miR-20a-5p inhibitor also decreased the protein levels of PPAR $\gamma, \mathrm{C} / \mathrm{EBP} \alpha$ and aP2 (Fig. 3G). These results suggested that inhibition of miR-20a-5p can restrain the adipogenic differentiation from BMSCs.

\section{KIf3 was a direct target gene of miR-20a-5p}

For further assessing the function of miR-20a-5p, it is important to determine which host mRNAs are regulated. We used TargetScanMouse (version $7.1 \mathrm{http}: / / \mathrm{www}$. targetscan.org/mmu_71/) to predict the candidate target genes of miR-20a-5p. Kdm6b and Tgfbr2 have been identified as direct targets of miR-20a-5p in our previous study (Zhou et al. 2015). In this report, we also found that there was a putative miR-20a-5p-binding site within the 3'-UTR of Klf3 (Fig. 4A). To determine whether Klf3 represent as a direct target of miR-20a-5p, we made the luciferase report constructs that contain the putative wildtype or mutant miR-20a-5p-binding sites within 3'UTR. We observed marked reduction in luciferase activity $(P<0.01)$ when co-transfecting the recombinant luciferase reporter constructs and miR-20a-5p mimics, compared to the cells co-transfected with the recombinant luciferase reporter constructs and NC; by contrast, mutation of the putative binding site rendered the construct loses the responsiveness to miR-20a-5p mimics (Fig. 4B). To confirm this, GFP repression assay was performed. GFP fluorescence was significantly reduced in HEK-293T cells transfected with GFP reporter constructs containing binding sites and miR-20a-5p mimics (Fig. 4C, D and E). Furthermore, overexpression of miR-20a-5p in BMSCs resulted in the downregulation of the mRNA and protein level of Klf3, and in contrast, miR-20a-5p inhibitor increased the mRNA and protein level of Klf3 (Fig. 4F and G). Taken together, these data suggest that Klf3 is a potential target of miR-20a-5p.

\section{KIf3 was potentially involved in miR-20a-5p- promoted adipocyte formation}

To investigate whether Klf3 is involved in miR-20a-5ppromoted adipogenic differentiation, we first tested if the silencing expression of Klf3 may have the similar proadipogenic effect as miR-20a-5p overexpression. BMSCs were transfected respectively with two Klf3 siRNAs or NC RNA (si-ctrl) for $48 \mathrm{~h}$. As shown in Fig. 5A, the mRNA level of Klf3 could be reduced by its siRNAs. Subsequently, Klf3 siRNAs obviously displayed stimulatory effect on the adipocyte formation from BMSCs (Fig. 5B and C). Silencing of Klf3 also significantly promoted the mRNA expression of Ppary, C/ebp $\alpha$ and aP2 (Fig. 5D), which phenocopied the effect of miR-20a-5p overexpression. Furthermore, we investigated whether Klf3 could counteract the pro-adipogenic function of miR-20a-5p. The expression construct pCDNA3.1-Klf3 (Klf3), which encoded the entire coding sequence of $K l f 3$, was made. We demonstrated the overexpression of Klf3 in BMSCs with RT-qPCR and immunoblotting (Fig. 5E). As shown in Fig. 5F and $\mathrm{G}$, overexpression of Klf3 abrogated miR-20a-5p-promoted adipogenesis. Consistently, the mRNA levels of adipogenic marker genes were decreased in the cells co-transfected with miR-20a-5p mimics and pCDNA3.1-Klf3, as compared to the cells co-transfected with miR-20a-5p mimics and vector (Fig. 5H). These results suggested that Klf3 is potentially involved in miR-20a-5p-promoted adipocyte formation.

\section{Discussion}

Adipogenic differentiation from BMSCs is regulated by multiple regulatory factors and signaling pathways. Upon induction of adipogenic factors, C/EBP $\beta$ and $\delta$ activate $\mathrm{C} / \mathrm{EBP} \alpha$ and $\mathrm{PPAR} \gamma$ to coordinate the expression of adipogenic genes characteristic of terminally differentiated adipocytes like aP2 (Kang \& Hata 2015). Moreover, emerging evidences suggest that miRNAs also regulate fate decisions of BMSCs to influence adipocyte differentiation (Scheideler 2016). In our previous report, we have shown that miR-20a-5p was upregulated in BMSCs and adipogenic cell lines 3T3-L1 and C3H10T1/2 3 days after adipogenic treatment (Zhou et al. 2015). In the current study, we further demonstrated the expression of miR-20a-5p was promoted gradually at the first 3 days during adipogenic differentiation from BMSCs, and its expression remained high level at the next 2 days (Fig. 1).

miR-20a-5p belongs to the miR-17-92 cluster that is highly expressed in various cancers (Hayashita et al. 2005, 

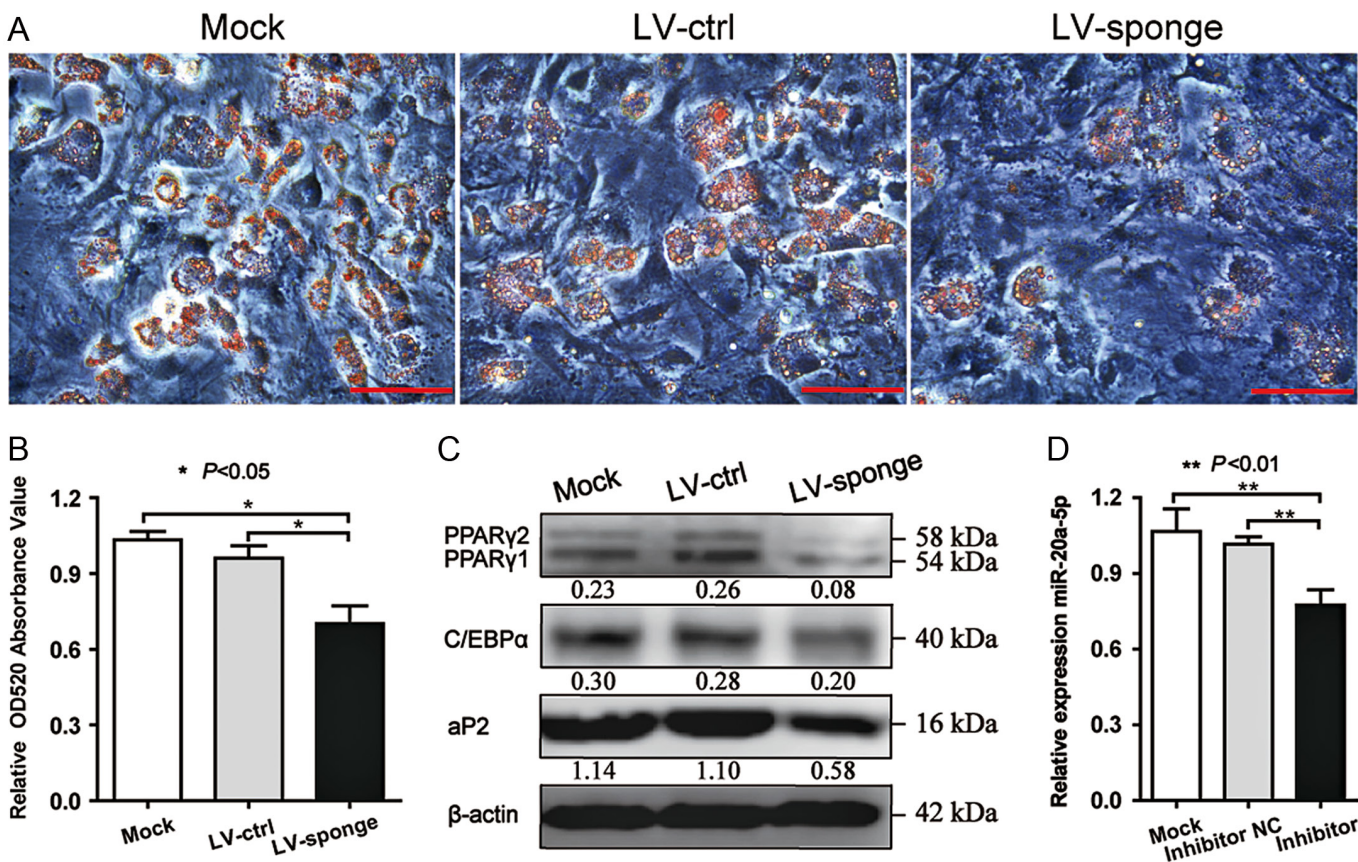

C
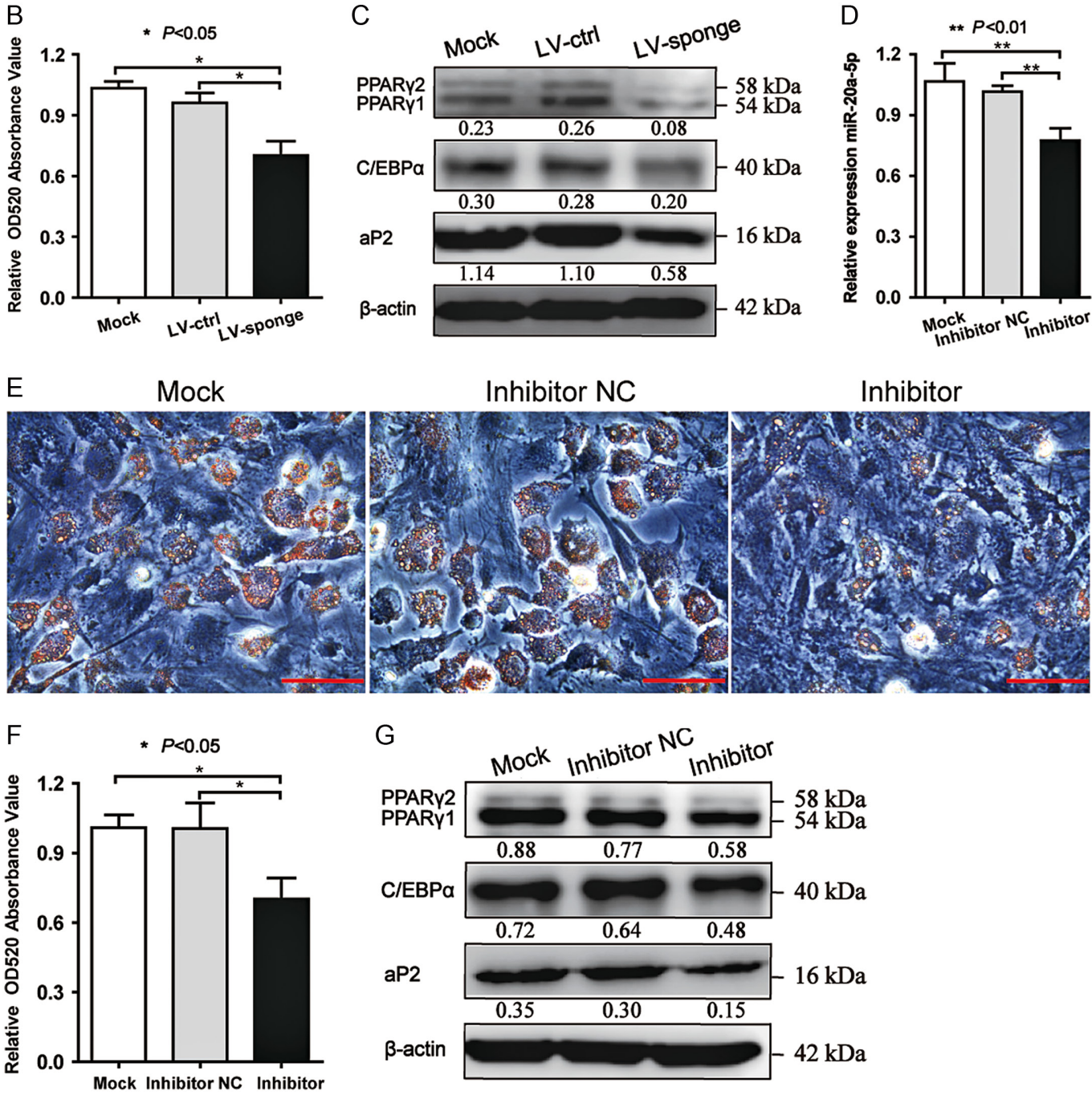

G

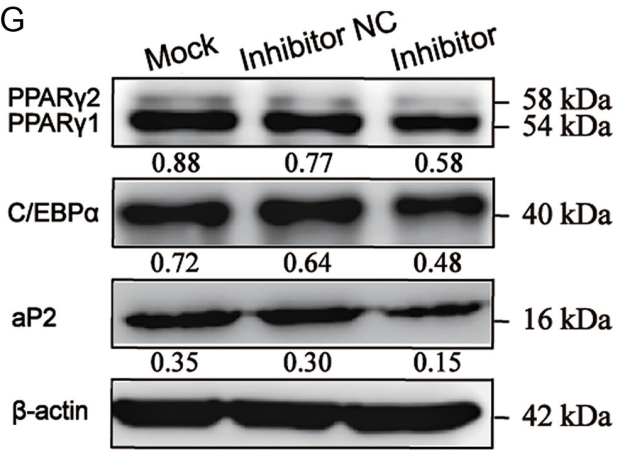

Figure 3

Inhibition of miR-20a-5p suppressed adipocyte formation from BMSCs. (A, B and C) BMSCs were infected with miR-20a-5p sponge lentivirus (LV-sponge) or control LV-sponge (LV-ctrl) for 2 days, and then cultured in AIM. After 5 days, the adipocyte-inducing BMSCs were labeled with Oil Red O, and representative images are shown (A). Oil Red O extracted with isopropanol was measured at OD520 (B). After 3 days, the adipocyte-inducing BMSCs were harvested, and the protein levels of PPAR, C/EBP $\alpha$ and aP2 were analyzed by immunoblotting (C). (D) Relative expression of miR-20a-5p was measured by RT-qPCR in BMSCs transfected with miR-20a-5p inhibitor or its negative control (Inhibitor NC) for 2 days. (E, F and G) BMSCs were transfected with miR-20a-5p inhibitor or inhibitor NC for 2 days, and then cultured in AIM. After 5 days, the adipocyte-inducing BMSCs were labeled with Oil Red O, and representative images were shown (E). Oil Red O extracted with isopropanol was measured at OD520 (F). After 3 days,

Immunoblotting for PPAR $\gamma$, C/EBP $\alpha$ and aP2 was performed (G). All data are from three separate experiments. All images were taken using a light microscope at 200x magnification, and the scale bars are presented at $100 \mu \mathrm{m}$. The value in $\mathrm{C}$ and $\mathrm{G}$ under each lane indicates the relative expression level of the putative target gene, which is represented by the intensity ratio between PPAR $\gamma$, C/EBP $\alpha$ or aP2 and $\beta$-actin bands in each lane; $\beta$-actin was used as the internal control. The values are mean \pm s.D. $(n=3), * P<0.05, * * P<0.01$, presented relative to NC group (ANOVA followed by Tukey's test was applied). 
A

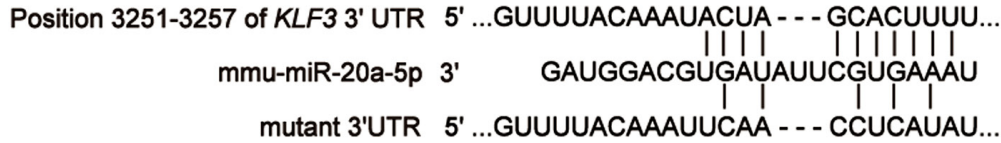

C
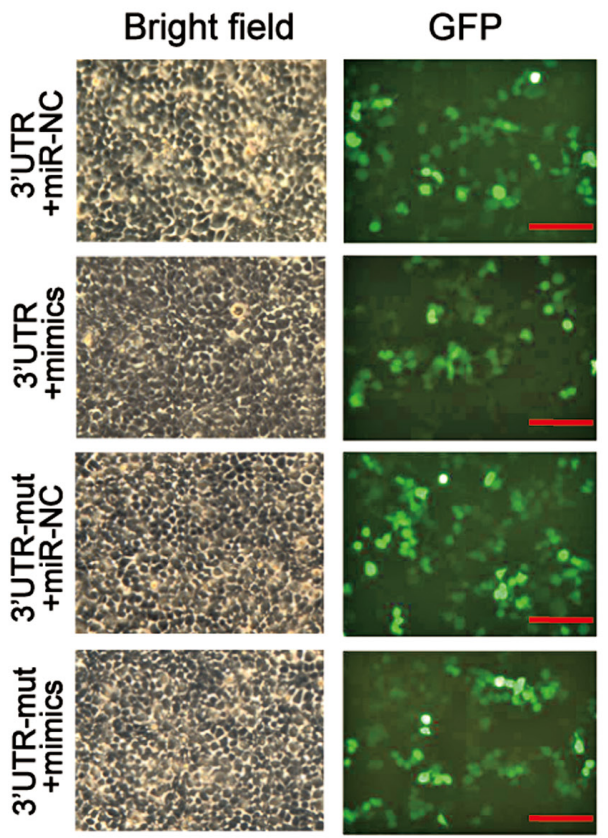

B

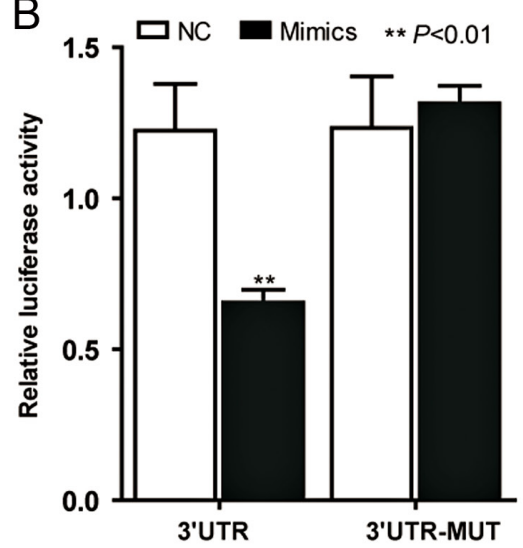

D

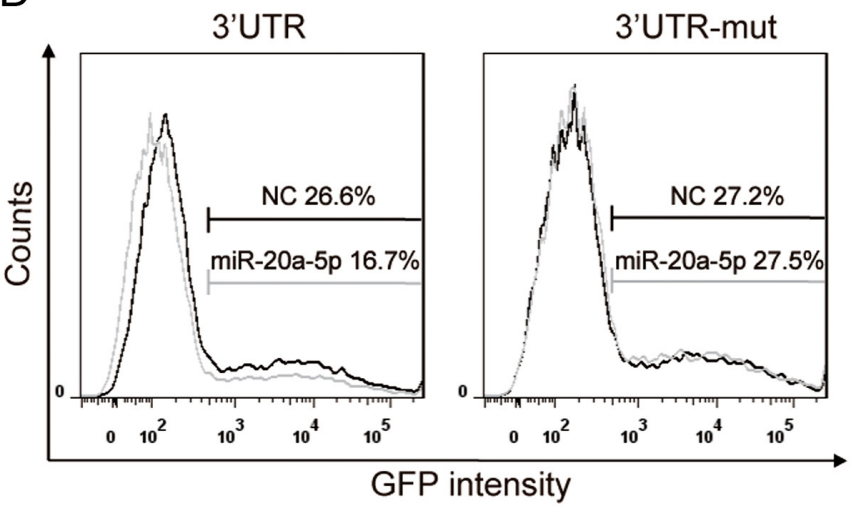

E

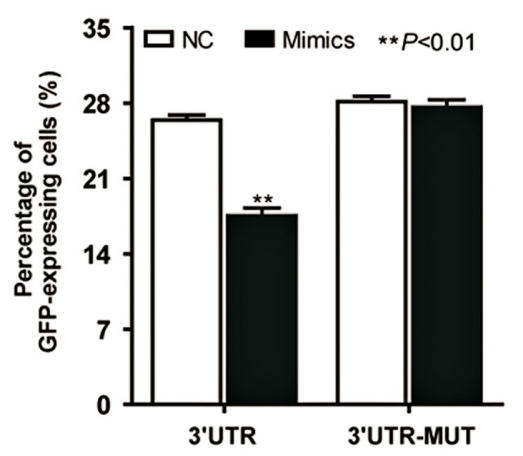

F

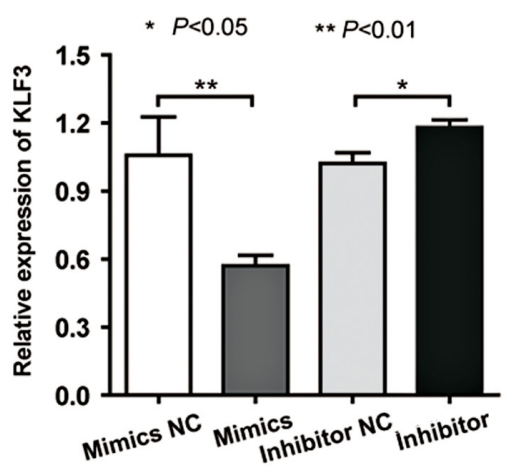

G

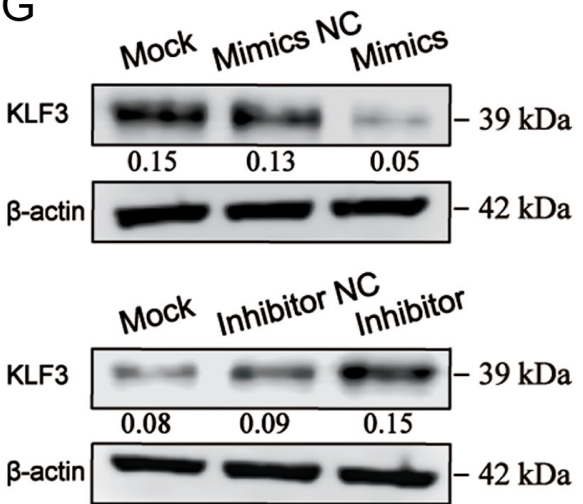

Figure 4

KIf3 was a candidate target gene of miR-20a-5p. (A) Sequence alignment of miR-20a-5p and its putative target site in the $3{ }^{\prime} U T R$ of $K I f 3$ mRNA. Mutation was made in the complementary sites for the seed region of miR-20a-5p as indicated. (B) Luciferase activity analysis. HEK-293T cells were transiently co-transfected with Renilla luciferase control vector pRL-TK, firefly luciferase reporter constructs containing either wild-type or mutant 3'UTR of KIf3, and either miR-20a-5p NC or mimics for $48 \mathrm{~h}$. Firefly luciferase activities were normalized to the activity of Renilla luciferase. (C and D) GFP repression assay. HEK-293T cells were transfected with the wild-type or mutant GFP construct, and either miR-20a-5p mimics or NC. GFP fluorescence was monitored by fluorescence microscope (C) and FCM analysis (D). (E) The statistical analysis of GFP repression rate for experiments in D is shown. (F) BMSCs were transfected with miR-20a-5p mimics, mimics NC, miR-20a-5p inhibitor or inhibitor NC. After $72 \mathrm{~h}$, the mRNA level of Klf3 was analyzed by RT-qPCR. (G) Western blot analysis for KIf3 in transfected cells in F is shown. The value under each lane indicates the relative expression level of the putative target gene, which is represented by the intensity ratio between KLF3 and $\beta$-actin bands in each lane, $\beta$-actin was used as the internal control. All data above are representative of at least three independent experiments. All image magnification is $100 \times$, and the scale bars are presented at $50 \mu \mathrm{m}$. The values are mean \pm S.D. $(n=3), * P<0.05, * * P<0.01$, presented vs control group (Student's $t$ test was applied in $\mathrm{F}$, and ANOVA followed by Tukey's test was applied in B and E). 
A

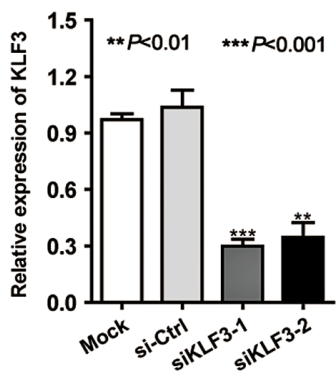

B

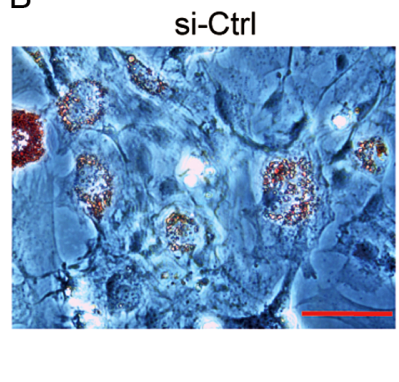

siKLF3-1

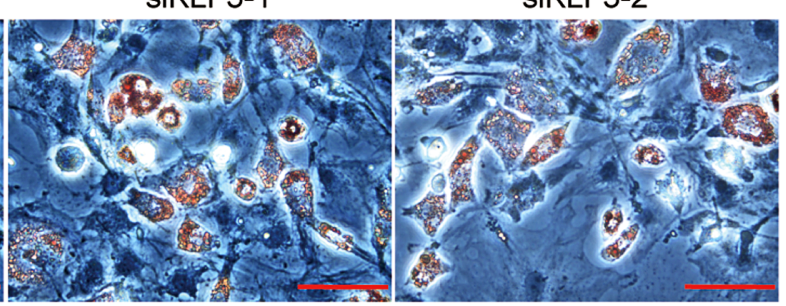

C

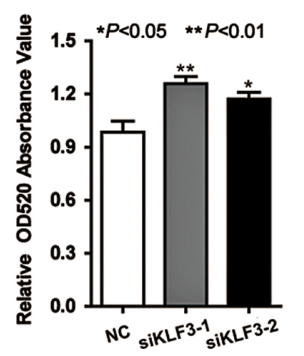

D

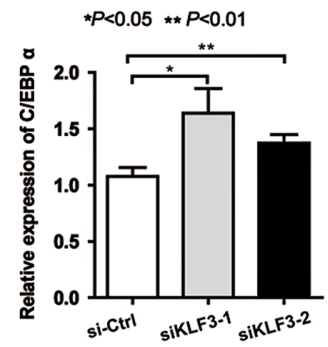

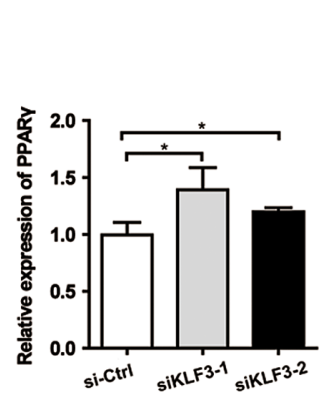
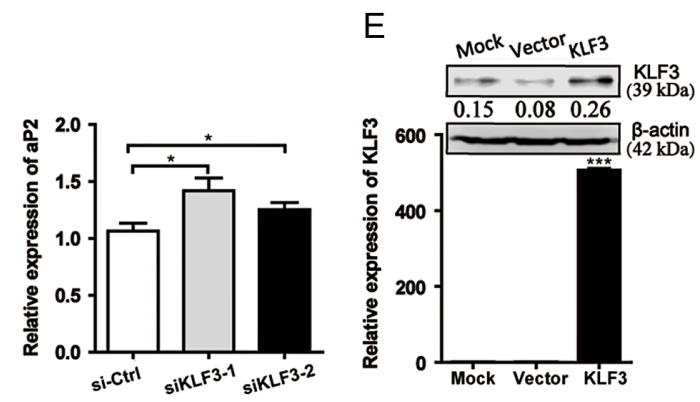

F
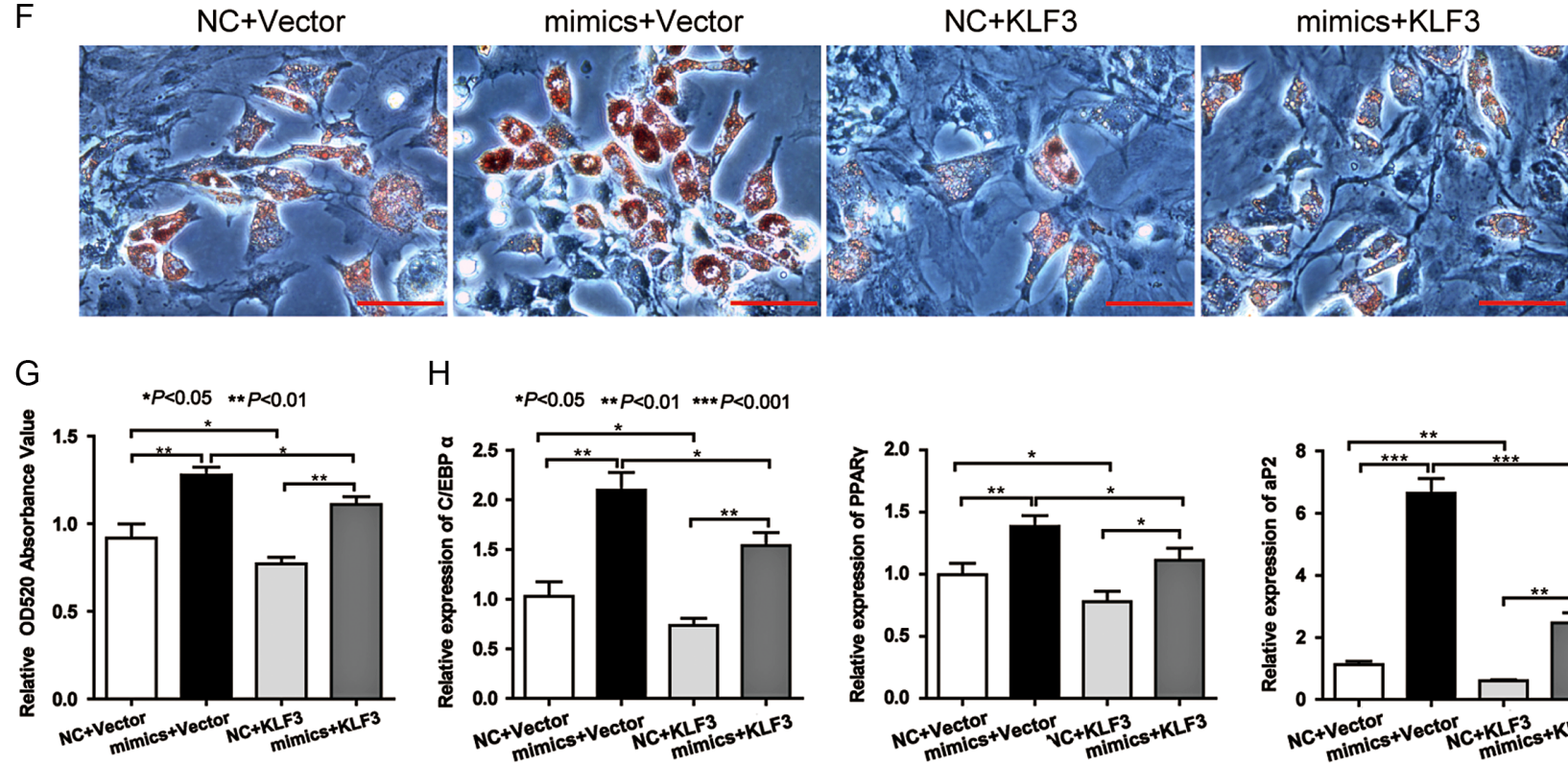

$\mathrm{H}$
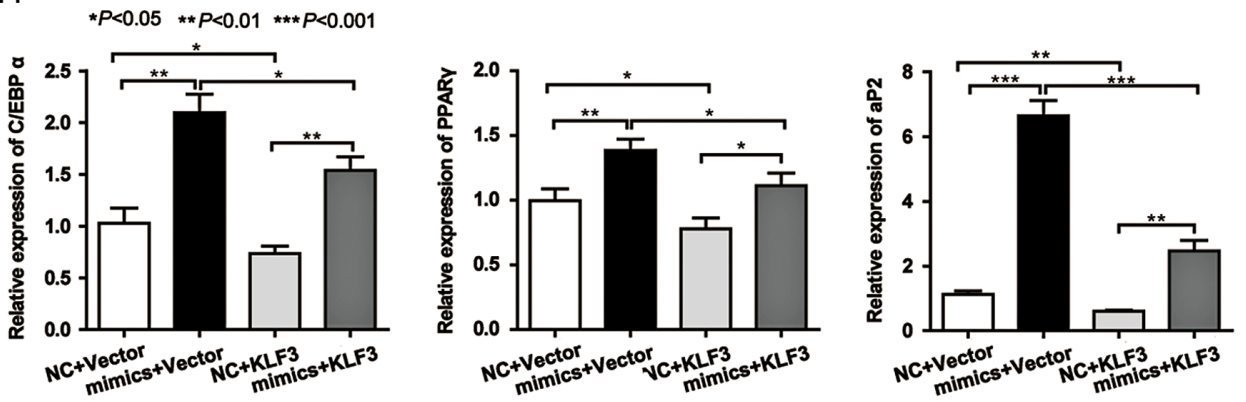

Figure 5

KIf3 was potentially involved in miR-20a-5p-promoted adipocyte formation. (A) RT-qPCR was used to monitor the expression of $K I f 348 \mathrm{~h}$ after transfection with KIf3 siRNAs (siKIf3) or NC RNA (si-Ctrl), Nono served as internal control. (B, C and D) BMSCs were transfected with si-Ctrl or siKlf3 for 2 days, and then cultured in AIM. After 5 days, the adipocyte-inducing BMSCs were stained with Oil Red O, and representative images are shown (B). Oil Red O extracted with isopropanol was measured at OD520 (C). RT-qPCR was performed to test the mRNA expression of adipocyte specific genes Clebp $\alpha$, Ppary and aP2 (D). (E) RT-qPCR and Western blotting were used to test the expression of Klf3 2 days after transfection with indicated expression construct, the value under each lane of Western blotting indicates the relative expression level of the putative target gene, which is represented by the intensity ratio between KLF3 and $\beta$-actin bands in each lane. NONO and $\beta$-actin served as internal controls respectively. $(F, G$ and $H)$ BMSCs were transfected with indicated RNA duplex for 1 day, and transfected with indicated expression vector for another day. Next, the cells were cultured in AIM. After 5 days, the BMSCs were stained with Oil Red O, and representative images are shown (F). Oil Red O extracted with isopropanol was measured at OD520 (G). The mRNA levels of Clebp $\alpha$, Ppary and aP2 were measured with RT-qPCR (H). All data are from three independent experiments. All image magnification is $200 x$, and the scale bars are presented at $100 \mu \mathrm{m}$. The values are mean \pm s.D. $(n=3), * P<0.05, * * P<0.01, * * * P<0.001$, presented vs control group (ANOVA followed by Tukey's test was applied). 
Takakura et al. 2008, Mu et al. 2009, Tsuchida et al. 2011). In recent years, several members of miR-17-92 cluster have been identified to be involved in the regulation of adipogenesis. miR-17-92 cluster accelerates adipocyte differentiation by negatively regulating tumor-suppressor Rb2/p130 in 3T3-L1 cells (Wang et al. 2008). miR-17-5p and miR-106a promote adipogenesis in human adipose-derived MSCs by targeting BMP2 (Li et al. 2013). We previously reported that miR-20a-5p increased adipogenesis in murine cell lines 3T3-L1, ST2 and C3H10T1/2 cells, which is dependent upon its inhibitory effects on KDM6B and TGF- $\beta$ signaling (Zhou et al. 2015). In the present study, we switched to the primary cultured BMSCs cell system to further clarify the function and mechanism of miR-20a-5p in adipogenic differentiation by gain- or loss-of-function studies. We found ectopic expression of miR-20a-5p, either by overexpression lentivirus or synthetic mimics, favored adipocyte differentiation; by contrast, inhibition of endogenous miR-20a-5p, either by sponge lentivirus or synthetic inhibitor, reduced adipocyte differentiation (Figs 2 and 3). These findings that miR-20a-5p affects adipogenesis of BMSCs coincided with our previous report (Zhou et al. 2015), and it suggests that miR-20a-5p have a pivotal role in adipogenic differentiation of BMSCs. We also found adipocyte differentiation could not be triggered by overexpression of miR-20a-5p without hormonal cocktail induction (data not shown), and we have previously shown that miR-20a-5p had no effect when the cells have differentiated after 3 days of adipogenic treatment (Zhou et al. 2015). All these results suggested that miR-20a-5p is a necessary adjunct to hormonal signals at the initial stage of adipogenic differentiation.

Next, it is important to identify target genes of miR-20a-5p for exploring the molecular mechanism underlying its function. Although $K d m 6 b$ and $T g f b r 2$ have been validated to involve in the regulation of adipogenesis by miR-20a-5p in our previous study (Zhou et al. 2015), there is no evidence to indicate that they play a regulatory role in initiate differentiation. In present study, we focused on whether there were target genes of miR-20a-5p at the early stage of adipocyte differentiation. Recently, numerous evidences suggest several KLF family members have important roles as immediate early regulators in adipogenesis. Oishi and coworkers reported that KLF5 expression is induced by $\mathrm{C} / \mathrm{EBP} \beta$ and $\delta$ and can directly bind to the endogenous $\mathrm{PPAR} \gamma$ promoter to modestly stimulate the activity of PPAR $\gamma$ promoter (Oishi et al. 2005). KLF4 modulates adipocyte formation by directly binding to the C/EBP $\beta$ promoter and together with Krox 20 cooperatively transactivates C/EBP $\beta$ (Birsoy et al. 2008). KLF2 directly binds to PPAR $\gamma 2$ promoter and inhibits the promoter activity to exert its suppressive role during adipogenesis (Banerjee et al. 2003). Overexpressing KLF7 decreases the differentiation of the human preadipocyte and inhibits glucose-induced insulin secretion in pancreatic $\beta$-cell line (Kawamura et al. 2006). Sue and coworkers reported that (I) the mRNA level of Klf3 was the highest at day 0 in 3T3-L1 preadipocytes and gradually diminished upon adipocyte differentiation; (II) overexpression of Klf3 could inhibit adipogenic differentiation of 3T3-L1 cells in vitro; (III) Klf3 can directly bind to the endogenous C/EBP $\alpha$ promoter and repress its activity (Sue et al. 2008). Considering that both $\mathrm{C} / \mathrm{EBP} \alpha$ and PPAR $\gamma$ can positively regulate the expression of one another in the early stage of adipogenic commitment (Mota de Sa et al. 2017), we conclude that Klf3 may suppress the activity of C/ebpo to reduce the expression of Ppary, and then, the expression of C/ebpo and Ppary will be decreased to a lower level in a negative feedback loop. Therefore, Klf3 was selected basing on its established inhibitory role in adipogenesis at the initial stage of differentiation, and we tried to identify whether it was a direct target of miR-20a-5p.

With the assistance of prediction programs, Klf3 was predicted to be a putative target because there was a targeting site for miR-20a-5p in its $3^{\prime}$ UTR (Fig. 4A). Subsequent luciferase assay and GFP repression experiments provided evidence that miR-20a-5p directly targeted the 3'UTR of Klf3 mRNA (Fig. 4B, C, D and E). We also showed that miR-20a-5p regulated Klf3 expression at the transcriptional level (Fig. 4F and G); in addition, the manipulate of miR-20a-5p mimics or inhibitor did not affect the protein level of two other KLFfamily members KLF5 and KLF7 (Supplementary Fig. 1, see section on supplementary data given at the end of this article), which indicates that Klf3 may be the only direct target of miR-20a-5p in KLF family members, and there may not be any compensatory changes of other KLF family members upon Klf3 upregulation or downregulation. Next, we found silencing of Klf3 expression had similar stimulatory effect on adipogenic differentiation compared with ectopic expression of miR-20a-5p (Fig. 5B, C and D), and overexpression of Klf3 could counteract the effect of increasing adipogenesis by miR-20a-5p (Fig. 5F, G and H); moreover, simultaneous overexpression of Klf3, Kdm6b and $T g f b r 2$ could be more effectively to neutralize the role of miR-20a-5p in promoting adipogenesis (Supplementary Fig. 2). Based on our experimental data, we propose the following model: after hormonal cocktail induction, the expression of miR-20a-5p is upregulated, resulting in a decrease of Klf3; then, the inhibition of C/EBPo is 
abrogated by the downregulation of KLF3; finally, PPAR $\gamma$ is activated to stimulate the expression of downstream adipogenic genes characteristic of mature adipocytes.

In summary, a series of present findings provided evidence that miR-20a-5p has a positive effect on adipogenic differentiation from BMSCs. We for the first time demonstrated miR-20a-5p promoted adipogenesis from BMSCs by directly targeting Klf3. Our findings expand our knowledge on the mechanism underlying the function of miR-20a-5p in adipogenesis and indicate the potential therapeutic application of miR-20a-5p in metabolic disorders caused by adipogenic dysregulation such as obesity.

\section{Supplementary data}

This is linked to the online version of the paper at https://doi.org/10.1530/ JME-17-0183.

\section{Declaration of interest}

The authors declare that there is no conflict of interest that could be perceived as prejudicing the impartiality of the research reported.

\section{Funding}

This work was supported by the National Natural Science Foundation of China (grant numbers 81501846, 81601864, 81672116 and 81472040); the Scientific Foundation of Tianjin Medical University (grant number 2015KYZM09) and Tianjin Municipal Natural Science Foundation of China (grant number 16JCYBJC26800).

\section{Author contribution statement}

Endong Zhu analyzed and interpreted the data and drafted the manuscript. Endong Zhu, Juanjuan Zhang, Jie Zhou, Hairui Yuan and Wei Zhao analyzed the data; Baoli Wang critically revised the manuscript and responsible for important intellectual content. All authors approved the final version for publication.

\section{References}

Arsenijevic T, Gregoire F, Delforge V, Delporte C \& Perret J 2012 Murine 3T3-L1 adipocyte cell differentiation model: validated reference genes for qPCR gene expression analysis. PLoS ONE 7 e37517. (https://doi.org/10.1371/journal.pone.0037517)

Banerjee SS, Feinberg MW, Watanabe M, Gray S, Haspel RL, Denkinger DJ, Kawahara R, Hauner H \& Jain MK 2003 The Krüppellike factor KLF2 inhibits peroxisome proliferator-activated receptor- $\gamma$ expression and adipogenesis. Journal of Biological Chemistry 278 2581-2584. (https://doi.org/10.1074/jbc.M210859200)

Birsoy K, Chen Z \& Friedman J 2008 Transcriptional regulation of adipogenesis by KLF4. Cell Metabolism 7 339-347. (https://doi. org/10.1016/j.cmet.2008.02.001)

Caplan AI \& Bruder SP 2001 Mesenchymal stem cells: building blocks for molecular medicine in the 21st century. Trends in Molecular Medicine 7 259-264. (https://doi.org/10.1016/S1471-4914(01)02016-0)
Guan X, Gao Y, Zhou J, Wang J, Zheng F, Guo F, Chang A, Li X \& Wang B 2015 miR-223 regulates adipogenic and osteogenic differentiation of mesenchymal stem cells through a C/EBPs/ miR-223/FGFR2 regulatory feedback loop. Stem Cells 33 1589-1600. (https://doi.org/10.1002/stem.1947)

Ha M \& Kim VN 2014 Regulation of microRNA biogenesis. Nature Reviews Molecular Cell Biology 15 509-524. (https://doi.org/10.1038/ nrm3838)

Hayashita Y, Osada H, Tatematsu Y, Yamada H, Yanagisawa K, Tomida S, Yatabe Y, Kawahara K, Sekido Y \& Takahashi T 2005 A polycistronic microRNA cluster, miR-17-92, is overexpressed in human lung cancers and enhances cell proliferation. Cancer Research 65 9628-9632. (https://doi.org/10.1158/0008-5472.CAN-05-2352)

Huang J, Zhao L, Xing L \& Chen D 2010 MicroRNA-204 regulates Runx2 protein expression and mesenchymal progenitor cell differentiation. Stem Cells 28 357-364. (https://doi.org/10.1002/stem.288)

Jeong B-C, Kang I-H \& Koh J-T 2014 MicroRNA-302a inhibits adipogenesis by suppressing peroxisome proliferator-activated receptor $\gamma$ expression. FEBS Letters 588 3427-3434. (https://doi. org/10.1016/j.febslet.2014.07.035)

Kang H \& Hata A 2015 The role of microRNAs in cell fate determination of mesenchymal stem cells: balancing adipogenesis and osteogenesis. BMB Reports 48 319. (https://doi.org/10.5483/BMBRep.2015.48.6.206)

Kang M, Yan L, Li Y, Zhang W, Wang H, Tang A \& Ou H 2013 Inhibitory effect of microRNA-24 on fatty acid-binding protein expression on 3T3-L1 adipocyte differentiation. Genetics and Molecular Research 12 5267-5277. (https://doi.org/10.4238/2013.November.7.1)

Kawamura Y, Tanaka Y, Kawamori R \& Maeda S 2006 Overexpression of Kruppel-like factor 7 regulates adipocytokine gene expressions in human adipocytes and inhibits glucose-induced insulin secretion in pancreatic beta-cell line. Molecular Endocrinology 20 844-856. (https://doi.org/10.1210/me.2005-0138)

Kinoshita M, Ono K, Horie T, Nagao K, Nishi H, Kuwabara Y, TakanabeMori R, Hasegawa K, Kita T \& Kimura T 2010 Regulation of adipocyte differentiation by activation of serotonin (5-HT) receptors 5-HT2AR and 5-HT2CR and involvement of microRNA-448-mediated repression of KLF5. Molecular Endocrinology 24 1978-1987. (https:// doi.org/10.1210/me.2010-0054)

Lefterova MI, Zhang Y, Steger DJ, Schupp M, Schug J, Cristancho A, Feng D, Zhuo D, Stoeckert CJ \& Liu XS 2008 PPAR $\gamma$ and C/EBP factors orchestrate adipocyte biology via adjacent binding on a genome-wide scale. Genes and Development 22 2941-2952. (https:// doi.org/10.1101/gad.1709008)

Li H, Li T, Wang S, Wei J, Fan J, Li J, Han Q, Liao L, Shao C \& Zhao RC 2013 miR-17-5p and miR-106a are involved in the balance between osteogenic and adipogenic differentiation of adipose-derived mesenchymal stem cells. Stem Cell Research 10 313-324. (https://doi. org/10.1016/j.scr.2012.11.007)

Mota de Sa P, Richard AJ, Hang H \& Stephens JM 2017 Transcriptional regulation of adipogenesis. Comprehensive Physiology 7 635-674. (https://doi.org/10.1002/cphy.c.160022)

Mu P, Han Y-C, Betel D, Yao E, Squatrito M, Ogrodowski P, de Stanchina E, D'Andrea A, Sander C \& Ventura A 2009 Genetic dissection of the miR-17 92 cluster of microRNAs in Myc-induced B-cell lymphomas. Genes and Development 23 2806-2811. (https:// doi.org/10.1101/gad.1872909)

Oishi Y, Manabe I, Tobe K, Tsushima K, Shindo T, Fujiu K, Nishimura G, Maemura K, Yamauchi T \& Kubota N 2005 Krüppel-like transcription factor KLF5 is a key regulator of adipocyte differentiation. Cell Metabolism 1 27-39. (https://doi.org/10.1016/j.cmet.2004.11.005)

Peng Y, Li H, Li X, Yu S, Xiang H, Peng J \& Jiang S 2016 MicroRNA-215 impairs adipocyte differentiation and co-represses FNDC3B and CTNNBIP1. International Journal of Biochemistry and Cell Biology 79 104-112. (https://doi.org/10.1016/j.biocel.2016.08.014)

Pittenger MF, Mackay AM, Beck SC, Jaiswal RK, Douglas R, Mosca JD, Moorman MA, Simonetti DW, Craig S \& Marshak DR 1999 
Multilineage potential of adult human mesenchymal stem cells. Science 284 143-147. (https://doi.org/10.1126/science.284.5411.143)

Rosen ED \& MacDougald OA 2006 Adipocyte differentiation from the inside out. Nature Reviews Molecular Cell Biology 7 885-896. (https:// doi.org/10.1038/nrm2066)

Sarjeant K \& Stephens JM 2012 Adipogenesis. Cold Spring Harbor Perspectives in Biology 4 a008417. (https://doi.org/10.1101/ cshperspect.a008417)

Scheideler M 2016 MicroRNAs in adipocyte formation and obesity. Best Practice and Research Clinical Endocrinology and Metabolism 30 653-664. (https://doi.org/10.1016/j.beem.2016.11.009)

Sue N, Jack BH, Eaton SA, Pearson RC, Funnell AP, Turner J, Czolij R, Denyer G, Bao S \& Molero-Navajas JC 2008 Targeted disruption of the basic Krüppel-like factor gene (Klf3) reveals a role in adipogenesis. Molecular and Cellular Biology 28 3967-3978. (https:// doi.org/10.1128/MCB.01942-07)

Sun T, Fu M, Bookout AL, Kliewer SA \& Mangelsdorf DJ 2009 MicroRNA let-7 regulates 3T3-L1 adipogenesis. Molecular Endocrinology 23 925-931. (https://doi.org/10.1210/me.2008-0298)

Takakura S, Mitsutake N, Nakashima M, Namba H, Saenko VA, Rogounovitch TI, Nakazawa Y, Hayashi T, Ohtsuru A \& Yamashita S 2008 Oncogenic role of miR-17-92 cluster in anaplastic thyroid cancer cells. Cancer Science 99 1147-1154. (https://doi. org/10.1111/j.1349-7006.2008.00800.x)

Tang QQ \& Lane MD 2012 Adipogenesis: from stem cell to adipocyte. Annual Review of Biochemistry 81 715-736. (https://doi.org/10.1146/ annurev-biochem-052110-115718)

Tian L, Zheng F, Li Z, Wang H, Yuan H, Zhang X, Ma Z, Li X, Gao X \& Wang B 2017 miR-148a-3p regulates adipocyte and osteoblast differentiation by targeting lysine-specific demethylase $6 \mathrm{~b}$. Gene 627 32-39 (https://doi.org/10.1016/j.gene.2017.06.002)

Tsuchida A, Ohno S, Wu W, Borjigin N, Fujita K, Aoki T, Ueda S, Takanashi M \& Kuroda M 2011 miR-92 is a key oncogenic component of the miR-17-92 cluster in colon cancer. Cancer Science 102 2264-2271. (https://doi.org/10.1111/j.1349-7006.2011.02081.x)

Wang Q, Li YC, Wang J, Kong J, Qi Y, Quigg RJ \& Li X 2008 miR-17-92 cluster accelerates adipocyte differentiation by negatively regulating tumor-suppressor Rb2/p130. PNAS 105 2889-2894. (https://doi. org/10.1073/pnas.0800178105)

Wang J, Guan X, Guo F, Zhou J, Chang A, Sun B, Cai Y, Ma Z, Dai C \& Li X 2013 miR-30e reciprocally regulates the differentiation of adipocytes and osteoblasts by directly targeting low-density lipoprotein receptor-related protein 6. Cell Death and Disease 4 e845. (https://doi.org/10.1038/cddis.2013.356)

Wu Z \& Wang S 2013 Role of Kruppel-like transcription factors in adipogenesis. Developmental Biology 373 235-243. (https://doi. org/10.1016/j.ydbio.2012.10.031)

Yeh W-C, Cao Z, Classon M \& McKnight SL 1995 Cascade regulation of terminal adipocyte differentiation by three members of the C/EBP family of leucine zipper proteins. Genes and Development 9 168-181. (https://doi.org/10.1101/gad.9.2.168)

Zhou J, Guo F, Wang G, Wang J, Zheng F, Guan X, Chang A, Zhang X, Dai C, Li S, et al. 2015 miR-20a regulates adipocyte differentiation by targeting lysine-specific demethylase $6 \mathrm{~b}$ and transforming growth factor- $\beta$ signaling. International Journal of Obesity 39 1282-1291. (https://doi.org/10.1038/ijo.2015.43)

Zhou J, Wang S, Qi Q, Yang X, Zhu E, Yuan H, Li X, Liu Y, Li X \& Wang B 2017 Nuclear factor IC reciprocally regulates adipocyte and osteoblast differentiation via control of canonical Wnt signaling. FASEB Journal 31 1939-1952. (https://doi.org/10.1096/ fj.201600975RR)

Zhu E, Wang X, Zheng B, Wang Q, Hao J, Chen S, Zhao Q, Zhao L, Wu Z \& Yin Z 2014 miR-20b suppresses Th17 differentiation and the pathogenesis of experimental autoimmune encephalomyelitis by targeting RORgammat and STAT3. Journal of Immunology 192 5599-5609. (https://doi.org/10.4049/jimmunol.1303488)

Zhu E, Yang Y, Zhang J, Li Y, Li C, Chen L \& Sun B 2016 Liraglutide suppresses obesity and induces brown fat-like phenotype via soluble guanylyl cyclase mediated pathway in vivo and in vitro. Oncotarget 7 81077. (https://doi.org/10.18632/oncotarget.13189)

Zuo P, Stanojević D, Colgan J, Han K, Levine M \& Manley J 1991 Activation and repression of transcription by the gap proteins hunchback and Krüppel in cultured Drosophila cells. Genes and development 5 254-264. (https://doi.org/10.1101/gad.5.2.254)

Received in final form 10 January 2018

Accepted 18 January 2018

Accepted Preprint published online 18 January 2018 